\begin{tabular}{|c|l|}
\hline Title & Spectral properties of a Dirac operator in the chiral quark soliton model \\
\hline Author(s) & A rai, A sao; Hay ashi, Kunimitsu; Sasaki, Itaru \\
\hline Citation & JOURNAL OF MA THEMATICAL PHY SICS, 46, 052306 \\
\hline https:/doi.org/L0.1063/1896388 \\
\hline Issue Date & 2005-05 \\
\hline Doc URL & http://hdl.handle.net/2115/5908 \\
\hline Rights & Copyright $\odot 2005$ A merican Institute of Physics \\
\hline Type & article \\
\hline File Information & JMP46-5.pdf \\
\hline
\end{tabular}

Instructions for use 


\title{
Spectral properties of a Dirac operator in the chiral quark soliton model
}

\author{
Asao Arai, ${ }^{a)}$ Kunimitsu Hayashi, and Itaru Sasaki \\ Department of Mathematics, Hokkaido University, Sapporo 060-0810, Japan
}

(Received 16 December 2004; accepted 1 March 2005; published online 20 April 2005)

We consider a Dirac operator $H$ acting in the Hilbert space $L^{2}\left(\mathbb{R}^{3} ; C^{4}\right) \otimes C^{2}$, which describes a Hamiltonian of the chiral quark soliton model in nuclear physics. The mass term of $H$ is a matrix-valued function formed out of a function $F: \mathbb{R}^{3} \rightarrow \mathbb{R}$, called a profile function, and a vector field $\mathbf{n}$ on $\mathbb{R}^{3}$, which fixes pointwise a direction in the isospin space of the pion. We first show that, under suitable conditions, $H$ may be regarded as a generator of a supersymmetry. In this case, the spectra of $H$ are symmetric with respect to the origin of R. We then identify the essential spectrum of $H$ under some condition for $F$. For a class of profile functions $F$, we derive an upper bound for the number of discrete eigenvalues of $H$. Under suitable conditions, we show the existence of a positive energy ground state or a negative energy ground state for a family of scaled deformations of $H$. A symmetry reduction of $H$ is also discussed. Finally a unitary transformation of $H$ is given, which may have a physical interpretation. (C) 2005 American Institute of Physics. [DOI: 10.1063/1.1896388]

\section{INTRODUCTION}

Let $\sigma_{j}(j=1,2,3)$ be the Pauli matrices,

$$
\sigma_{1}:=\left(\begin{array}{cc}
0 & 1 \\
1 & 0
\end{array}\right), \quad \sigma_{2}:=\left(\begin{array}{cc}
0 & -i \\
i & 0
\end{array}\right), \quad \sigma_{3}:=\left(\begin{array}{cc}
1 & 0 \\
0 & -1
\end{array}\right)
$$

and

$$
\alpha_{j}:=\left(\begin{array}{cc}
\sigma_{j} & 0_{2} \\
0_{2} & -\sigma_{j}
\end{array}\right) \quad(j=1,2,3), \quad \beta:=\left(\begin{array}{ll}
0_{2} & 1_{2} \\
1_{2} & 0_{2}
\end{array}\right),
$$

where $0_{2}$ and $1_{2}$ are the $2 \times 2$ zero matrix and the $2 \times 2$ identity matrix, respectively. The matrix

$$
\gamma_{5}:=-i \alpha_{1} \alpha_{2} \alpha_{3}
$$

is Hermitian with $\gamma_{5}^{2}=1_{4}$ (the $4 \times 4$ identity matrix) satisfying the following relations:

$$
\left[\alpha_{j}, \gamma_{5}\right]=0(j=1,2,3), \quad\left\{\beta, \gamma_{5}\right\}=0,
$$

where $[A, B]:=A B-B A$ and $\{A, B\}:=A B+B A$. We set

$$
\boldsymbol{\sigma}:=\left(\sigma_{1}, \sigma_{2}, \sigma_{3}\right), \quad \boldsymbol{\alpha}:=\left(\alpha_{1}, \alpha_{2}, \alpha_{3}\right) .
$$

For objects $\mathbf{A}=\left(A_{1}, A_{2}, A_{3}\right)$ and $\mathbf{B}=\left(B_{1}, B_{2}, B_{3}\right)$ such that the products $A_{j} B_{j}(j=1,2,3)$ and their sum are defined, we write $\mathbf{A} \cdot \mathbf{B}:=\sum_{j=1}^{3} A_{j} B_{j}$.

We consider a Dirac operator acting in the Hilbert space

\footnotetext{
${ }^{a)}$ Author to whom correspondence should be addressed. Electronic mail: arai@math.sci.hokudai.ac.jp 


$$
\mathcal{H}:=L^{2}\left(\mathbb{R}^{3} ; \mathrm{C}^{4}\right) \otimes \mathrm{C}^{2},
$$

where $L^{2}\left(\mathbb{R}^{3} ; C^{4}\right)$ is the Hilbert space of $C^{4}$-valued square integrable functions on $\mathbb{R}^{3}$. Let $\nabla:=\left(D_{1}, D_{2}, D_{3}\right)$ with $D_{j}$ the generalized partial differential operator in the variable $x_{j}$, the $j$ th component of $\mathbf{x}=\left(x_{1}, x_{2}, x_{3}\right) \in \mathbb{R}^{3}$. Then the free Dirac operator with mass zero is defined by

$$
H_{0}:=-i \boldsymbol{\alpha} \cdot \nabla \otimes 1_{2}
$$

acting in $\mathcal{H}$. To introduce a perturbation to $H_{0}$, let $F: \mathbb{R}^{3} \rightarrow \mathbb{R}$ be Borel measurable and finite almost everywhere (a.e.) in $\mathrm{R}^{3}$ and set

$$
U_{F}:=\cos F+i \gamma_{5} \otimes \boldsymbol{\tau} \cdot \mathbf{n} \sin F,
$$

where $\boldsymbol{\tau}:=\left(\tau_{1}, \tau_{2}, \tau_{3}\right)$ with $\tau_{j}:=\sigma_{j}(j=1,2,3), \mathbf{n}:=\left(n_{1}, n_{2}, n_{3}\right)$ with $n_{j}$ a real-valued measurable function on $\mathbb{R}^{3}$ such that

$$
|\mathbf{n}(\mathbf{x})|^{2}=1 \text {, a.e. } \mathbf{x} \in \mathbb{R}^{3} .
$$

Let $M>0$ be a constant. Then, by the second relation in (1.4), $M\left(\beta \otimes 1_{2}\right) U_{F}$ is a bounded selfadjoint operator on $\mathcal{H}$. Hence, by the Kato-Rellich theorem, the operator

$$
H:=H_{0}+M\left(\beta \otimes 1_{2}\right) U_{F}
$$

is self-adjoint with domain $D(H)=D\left(H_{0}\right)$. This is the Dirac operator we consider in this paper. The operator $H$ appears as the Hamiltonian of the so-called the chiral quark soliton model in nuclear physics (e.g., Ref. 1 and references therein). In this context, $M$ and

$$
\Phi_{F}:=\cos F+i \sin F \otimes \boldsymbol{\tau} \cdot \mathbf{n}
$$

( $U_{F}$ with $\gamma_{5}$ replaced by $1_{4}$ ) denote the mass of a quark and the pion field, respectively, and $F$ is called a profile function. The Dirac operator $H$ is not only physically important, but also may have interests from purely mathematical points of view. As far as we know, no mathematically rigorous analysis has been made on the Dirac operator $H$ (a study of a Dirac operator with a variable mass is given in Ref. 2, but, in that paper, the mass is a scalar function and the point there is to establish self-adjointness of such a Dirac opeartor in cases where the Kato-Rellich theorem is no longer applicable to it; in this sense Ref. 2 does not bear upon the topics of the present paper).

The present paper is organized as follows. In Sec. II, we show that the Dirac operator $H$ can be regarded as a generator of a supersymmetry, and describe its implications on the spectra of $H$. In Sec. III we identify the essential spectrum of $H$. We also derive an upper bound for the number of discrete eigenvalues of $H$. In particular, for a class of $F$ and $\mathbf{n}$, the absence of discrete eigenvalues of $H$ is proven. Sections IV and V are concerned with existence of discrete eigenvalues of $H$. In Sec. IV we introduce a concept of a positive energy ground state and that of a negative energy ground state of $H$ and show, under some condition for $F$, that a scaled deformation of $H$ has a positive energy ground state or a negative ground state. In Sec. V we discuss a symmetry reduction of $H$ to smaller mutually orthogonal closed subspaces which are indexed by triples $(\ell, s, t) \in \mathbb{Z} \times\{ \pm 1\} \times\{ \pm 1\}$, where $\ell$ denote an eigenvalue of the third component of the angular momentum operator, $s / 2$ the spin of the quark and $t / 2$ the isospin of the pion. We prove that, under suitable conditions, each reduced part of $H$ or its scaled version has a discrete positive ground state or a discrete negative ground state. In the last section we present a unitary transformation which brings $H$ to a Dirac operator with a magnetic moment.

\section{SUPERSYMMETRIC ASPECTS}

In this section we assume the following.

Hypothesis (I): Each $n_{j}(j=1,2,3)$ is continuously differentiable on $\mathbb{R}^{3}$ and

$$
\left(n_{1}(\mathbf{x}), n_{2}(\mathbf{x})\right) \neq(0,0), \quad \mathbf{x} \in \mathbb{R}^{3} .
$$


Let

$$
\xi(\mathbf{x}):=\frac{\left(\tau_{1} n_{2}(\mathbf{x})-\tau_{2} n_{1}(\mathbf{x})\right)}{\sqrt{n_{1}(\mathbf{x})^{2}+n_{2}(\mathbf{x})^{2}}}, \quad \mathbf{x} \in \mathbb{R}^{3} .
$$

Then $\xi(\mathbf{x})^{2}=1, \mathbf{x} \in \mathbb{R}^{3}$. For all $\mathbf{x} \in \mathbb{R}^{3}$, we can define a matrix tensor

$$
\Gamma(\mathbf{x}):=\alpha_{1} \alpha_{2} \alpha_{3} \beta \otimes \xi(\mathbf{x})
$$

acting on $\mathrm{C}^{4} \otimes \mathrm{C}^{2}$. It is easy to see that $\Gamma(\mathbf{x})$ is self-adjoint with $\Gamma(\mathbf{x})^{2}=I$ ( $I$ denotes identity). By the natural identification $\mathcal{H}=L^{2}\left(\mathbb{R}^{3} ; \mathrm{C}^{4} \otimes \mathrm{C}^{2}\right)$, we denote the multiplication operator by the matrixtensor valued function $\Gamma(\cdot)$ by the same symbol $\Gamma$. Then $\Gamma$ is self-adjoint and unitary on $\mathcal{H}$.

Proposition 2.1: Suppose that Hypothesis (I) holds and $\xi(\mathbf{x})$ is a constant matrix. Then, for all $\psi \in D(H), \Gamma \psi \in D(H)$ and

$$
\{\Gamma, H\} \psi=0, \quad \psi \in D(H) .
$$

Proof: By direct computations, we have

$$
\left\{\alpha_{1} \alpha_{2} \alpha_{3} \beta, \alpha_{j}\right\}=0 \quad(j=1,2,3), \quad\{\xi(\mathbf{x}), \boldsymbol{\tau} \cdot \mathbf{n}(\mathbf{x})\}=0 .
$$

Using these relations and the constancy of $\xi(\cdot)$, we see that, for all $\psi \in D(H)=D\left(H_{0}\right), \Gamma \psi \in D\left(H_{0}\right)$ and $H_{0} \Gamma \psi=-\Gamma H_{0} \psi$. Similarly, using (2.5) and $\left[\alpha_{1} \alpha_{2} \alpha_{3} \beta, \beta \gamma_{5}\right]=0$, we see that $\{M(\beta$ $\left.\left.\otimes 1_{2}\right) U_{F}, \Gamma\right\} \psi=0$. Thus (2.4) follows.

Proposition 2.1 shows that the Dirac operator $H$ may be regarded as a generator of a supersymmetry, i.e., a supercharge with respect to $\Gamma$ (e.g., p. 140 in Ref. 3).

For a self-adjoint operator $T$, we denote by $\sigma(T)$ [respectively, $\sigma_{\mathrm{p}}(T)$ ] the spectrum of $T$ (respectively, the point spectrum of $T$ ). The discrete spectrum of $T$ (the set of isolated eigenvalues of $T$ with finite multiplicity) is denoted $\sigma_{\mathrm{d}}(T)$.

Theorem 2.2: Suppose that Hypothesis (I) holds and $\xi(\mathbf{x})$ is a constant matrix. Then

(i) $\sigma(H)$ is symmetric with respect to the origin of $\mathbb{R}$, i.e., if $\lambda \in \sigma(H)$, then $-\lambda \in \sigma(H)$.

(ii) $\quad \sigma_{\#}(H)(\#=\mathrm{p}, \mathrm{d})$ is symmetric with respect to the origin of $\mathbb{R}$. The multiplicity $\lambda \in \sigma_{\#}(H)$ coincides with that of $-\lambda \in \sigma_{\#}(H)$.

Proof: By Proposition 2.1 we have $\Gamma H \Gamma^{-1}=-H$ (the unitary equivalence of $H$ and $-H$ ). This implies the desired results.

Remark 2.1: The properties stated in Theorem 2.2 may differ from spectral properties of the usual Dirac operator $H_{0}+M \beta+V$, where $V$ is a scalar potential.

\section{THE ESSENTIAL SPECTRUM AND FINITENESS OF THE DISCRETE SPECTRUM OF $H$}

\section{A. Structure of the spectrum of $H$}

For a self-adjoint operator $T$, we denote by $\sigma_{\text {ess }}(T)$ the essential spectrum of $T$.

Theorem 3.1: Suppose that

$$
\lim _{|\mathbf{x}| \rightarrow \infty} F(\mathbf{x})=0
$$

Then

$$
\begin{gathered}
\sigma_{\text {ess }}(H)=(-\infty,-M] \cup[M, \infty), \\
\sigma_{\mathrm{d}}(H) \subset(-M, M) .
\end{gathered}
$$

Proof: We write $H=H_{0}+M\left(\beta \otimes I_{2}\right)+V$ with $V:=M\left(\beta \otimes I_{2}\right)\left(U_{F}-I\right)$. We have $\|V(x)\| \leqslant M(\mid 1$ $-\cos F(x)|+| \sin F(x) \mid) \rightarrow 0(|x| \rightarrow \infty)$. Hence we can apply Theorem 4.7, Remark 2 on p. 117 in Ref. 
3 to $H$ to obtain (3.2). This implies (3.3).

\section{B. Bound for the number of discrete eigenvalues of $\boldsymbol{H}$}

Assume (3.1). Then, by Theorem 3.1, we can define the number of discrete eigenvalues of $H$ counting multiplicities,

$$
N_{H}:=\operatorname{dim} \operatorname{Ran} E_{H}((-M, M)),
$$

where $E_{H}$ is the spectral measure of $H$ and $\operatorname{Ran} E_{H}((-M, M))$ means the range of $E_{H}((-M, M))$. To estimate an upper bound for $N_{H}$, we introduce a hypothesis for $F$ and $\mathbf{n}$.

\section{Hypothesis (II):}

(i) The functions $F$ and $n_{j}(j=1,2,3)$ are continuously differentiable on $\mathbb{R}^{3}$.

(ii) The functions $D_{j} F$ and $D_{j} n_{k}(j, k=1,2,3)$ are bounded on $\mathbb{R}^{3}$.

Under this assumption, we can define

$$
V_{F}(\mathbf{x}):=\sqrt{|\nabla F(\mathbf{x})|^{2}+\sum_{k=1}^{3}\left|\nabla n_{k}(\mathbf{x})\right|^{2} \sin ^{2} F(\mathbf{x})}, \quad \mathbf{x} \in R^{3} .
$$

Theorem 3.2: Assume (3.1) and Hypothesis (II). Suppose that

$$
C_{F}:=\int_{\mathbb{R}^{6}} \frac{V_{F}(\mathbf{x}) V_{F}(\mathbf{y})}{|\mathbf{x}-\mathbf{y}|^{2}} \mathrm{~d} \mathbf{x} \mathrm{d} \mathbf{y}<\infty .
$$

Then $N_{H}$ is finite with

$$
N_{H} \leqslant \frac{M^{2} C_{F}}{2 \pi^{2}} .
$$

To prove this theorem we present a general lemma. Let $\mathcal{K}$ be a complex Hilbert space and $\mathrm{B}(\mathcal{K})$ be the Banach space of bounded linear operators on $\mathcal{K}$. Let $V: \mathbb{R}^{d} \rightarrow \mathrm{B}(\mathcal{K})(d \in \mathbb{N})$ be a measurable function. The function $V$ defines a unique multiplication operator acting in the Hilbert space $L^{2}\left(\mathbb{R}^{d} ; \mathcal{K}\right)$ of $\mathcal{K}$-valued square integrable functions on $\mathbb{R}^{d}$. We denote it by the same symbol $V$. We assume the following ( $\Delta$ is the $d$-dimensional generalized Laplacian):

(V.1) $D\left((-\Delta)^{1 / 2}\right) \subset D\left(|V|^{1 / 2}\right) \cap D\left(\left|V^{*}\right|^{1 / 2}\right)$ and the form sum

$$
L_{0}:=-\Delta \dot{+}\left(\begin{array}{cc}
-|V| & 0 \\
0 & -\left|V^{*}\right|
\end{array}\right)
$$

acting in $\oplus^{2} L^{2}\left(\mathbb{R}^{d} ; \mathcal{K}\right)$ with form domain $D\left((-\Delta)^{1 / 2}\right)$ defines a unique self-adjoint operator bounded from below. Moreover, $\sigma_{\text {ess }}\left(L_{0}\right) \subset[0, \infty)$.

(V.2) The operator

$$
L:=-\Delta+\left(\begin{array}{cc}
0 & V^{*} \\
V & 0
\end{array}\right)
$$

acting in $\oplus^{2} L^{2}\left(\mathbb{R}^{d} ; \mathcal{K}\right)$ is self-adjoint on $D(\Delta)$, bounded from below, and $\sigma_{\text {ess }}(L) \subset[0, \infty)$.

For a self-adjoint operator $A$, we denote by $N_{-}(A)$ the number of negative eigenvalues of $A$ counting multiplicities.

Lemma 3.3: Assume (V.1) and (V.2). Then $N_{-}(L) \leqslant N_{-}\left(L_{0}\right)$.

Proof: Let 


$$
Q:=\left(\begin{array}{cc}
0 & V^{*} \\
V & 0
\end{array}\right)
$$

Then $Q$ is self-adjoint and

$$
Q^{2}=\left(\begin{array}{cc}
|V|^{2} & 0 \\
0 & \left|V^{*}\right|^{2}
\end{array}\right)
$$

which implies that

$$
|Q|=\left(\begin{array}{cc}
|V| & 0 \\
0 & \left|V^{*}\right|
\end{array}\right) .
$$

It is obvious that $Q \geqslant-|Q|$. Hence $L \geqslant L_{0}$. This inequality and the min-max principle (e.g., Theorem XIII.1, Problem 1 in Ref. 4) imply the inequality $N_{-}(L) \leqslant N_{-}\left(L_{0}\right)$.

Proof of Theorem 3.2: We note that $H$ has the operator matrix representation

$$
H=H_{0}+M\left(\begin{array}{cc}
0 & \Phi_{F}^{*} \\
\Phi_{F} & 0
\end{array}\right)
$$

where $\Phi_{F}$ is defined by (1.11). Hence

$$
H^{2}=L(F)+M^{2}
$$

with

$$
L(F):=-\Delta+M\left(\begin{array}{cc}
0 & W_{F}^{*} \\
W_{F} & 0
\end{array}\right),
$$

where $W_{F}:=i \boldsymbol{\sigma} \cdot\left(\nabla \Phi_{F}\right)$. Note that, by Hypothesis (II) (ii), the second term on the right-hand side of (3.10) is a bounded self-adjoint operator and hence $L(F)$ is self-adjoint with $D(L(F))=D(\Delta)$. By direct computations, we have

$$
W_{F}(\mathbf{x})^{*} W_{F}(\mathbf{x})=W_{F}(\mathbf{x}) W_{F}(\mathbf{x})^{*}=|\nabla F(\mathbf{x})|^{2}+\sum_{j=1}^{3}\left|\nabla n_{j}(\mathbf{x})\right|^{2} \sin ^{2} F(\mathbf{x}),
$$

where we have used (1.9). Hence $\left|W_{F}\right|=\left|W_{F}^{*}\right|=V_{F}$. Let $L_{0}(F):=-\Delta-M V_{F}$. By Theorem 3.1, $\sigma_{\text {ess }}(L(F))=[0, \infty)$. Condition (3.6) implies that $V_{F}$ is a potential in the Rollnik class (p. 170 in Ref. 5). Hence it follows from Example 7, p. 118 in Ref. 4 and Weyl's essential spectrum theorem (Theorem XIII.14, p. 112 in Ref. 4) that $\sigma_{\text {ess }}\left(L_{0}(F)\right)=\sigma_{\text {ess }}(-\Delta)=[0, \infty)$. Therefore the assumption of Lemma 3.3 with $L=L(F)$ and $L_{0}=L_{0}(F)$ is satisfied. Hence $N_{-}(L(F)) \leqslant N_{-}\left(L_{0}(F)\right)$. It is well known that $N_{-}\left(L_{0}(F)\right) \leqslant 8 M^{2} C_{F} /(4 \pi)^{2}$ (Theorem XIII.10 in Ref. 4), where the factor $8=\operatorname{dim} C^{4}$ $\otimes C^{2}$. On the other hand, by the spectral theorem, $N_{H} \leqslant N_{-}\left(L_{F}\right)$. Thus (3.7) follows.

Theorem 3.2 implies the absence of discrete eigenvalues of $H$ for $F$ 's such that the Rollnik norm of $M V_{F}$ is sufficiently small.

Corollary 3.4: Assume (3.1) and Hypothesis (II). Let $M^{2} C_{F}<2 \pi^{2}$. Then $\sigma_{\mathrm{d}}(H)=\emptyset$.

\section{EXISTENCE OF DISCRETE GROUND STATES}

For a self-adjoint operator $A$ bounded from below, we set

$$
E_{0}(A):=\inf \sigma(A)
$$

If $E_{0}(A) \in \sigma_{\mathrm{p}}(A)$, then we say that $A$ has a ground state and we call a nonzero vector in $\operatorname{ker}(A$ $\left.-E_{0}(A)\right)$ a ground state of $A$. If $E_{0}(A) \in \sigma_{\mathrm{d}}(A)$, then we say that $A$ has a discrete ground state.

Definition 4.1: Let 


$$
E_{0}^{+}(H):=\inf (\sigma(H) \cap[0, \infty)), \quad E_{0}^{-}(H):=\sup (\sigma(H) \cap(-\infty, 0]) .
$$

If $E_{0}^{+}(H)$ [respectively, $\left.E_{0}^{-}(H)\right]$ is an eigenvalue of $H$, then we say that $H$ has a positive (respectively, negative) energy ground state and we call a nonzero vector in $\operatorname{ker}\left(H-E_{0}^{+}(H)\right)$ [respectively, $\left.\operatorname{ker}\left(H-E_{0}^{-}(H)\right)\right]$ a positive (respectively, negative) energy ground state of $H$. If $E_{0}^{+}(H)$ [respectively, $\left.E_{0}^{-}(H)\right]$ is a discrete eigenvalue of $H$, then we say that $H$ has a discrete positive (respectively, negative) energy ground state.

Remark 4.1: If the spectrum of $H$ is symmetric with respect to the origin of $\mathrm{R}$ as in Theorem 2.2, then $E_{0}^{+}(H)=-E_{0}^{-}(H)$, and $H$ has a positive energy ground state if and only if it has a negative energy ground state.

We assume Hypothesis (II). Then the operators

$$
S_{ \pm}(F):=-\Delta \pm M\left(D_{3} \cos F\right)=-\Delta \mp M\left(D_{3} F\right) \sin F
$$

are self-adjoint with $D\left(S_{ \pm}(F)\right)=D(\Delta)$ and bounded from below.

Theorem 4.2: Assume Hypothesis (II) and (3.1). Suppose that $E_{0}\left(S_{+}(F)\right)<0$ or $E_{0}\left(S_{-}(F)\right)$ $<0$. Then $H$ has a discrete positive energy ground state or a discrete negative ground state.

Proof: For each $f \in D(\Delta)$ and $u \in \mathrm{C}^{2}$ with $\|u\|=1$, we define

$$
\psi_{f}^{+}:=(f \otimes u, 0, \text { if } \otimes u, 0) \in \mathcal{H}, \quad \psi_{f}^{-}:=(0, f \otimes u, 0, \text { if } \otimes u) \in \mathcal{H} .
$$

Then we have

$$
\left\langle\psi_{f}^{ \pm}, L(F) \psi_{f}^{ \pm}\right\rangle=2\left\langle f, S_{ \pm}(F) f\right\rangle .
$$

In the case where $E_{0}\left(S_{+}(F)\right)<0$, there exists a unit vector $f \in D(\Delta)$ such that $\left\langle f, S_{+}(F) f\right\rangle<0$. Hence $\left\langle\psi_{f}^{+}, L(F) \psi_{f}^{+}\right\rangle<0$. By Theorem 3.1 and the spectral theorem, we have

$$
\sigma_{\text {ess }}(L(F))=[0, \infty) .
$$

Thus, by the min-max principle, $L(F)$ has a discrete ground state. Similarly, in the case where $E_{0}\left(S_{-}(F)\right)<0$ too, $L(F)$ has a discrete ground state. This implies that $H$ has a discrete positive energy ground state or a discrete negative ground state.

To construct examples of $F$ satisfying the conditions as stated in Theorem 4.2, we consider a scaling. For a constant $\varepsilon>0$ and a function $f$ on $\mathbb{R}^{d}$, we define a function $f_{\varepsilon}$ on $\mathbb{R}^{d}$ by

$$
f_{\varepsilon}(x):=f(\varepsilon x), \quad x \in \mathbb{R}^{d} .
$$

Lemma 4.3: Let $V: \mathbb{R}^{d} \rightarrow \mathbb{R}$ be in $L_{\mathrm{loc}}^{2}\left(\mathbb{R}^{d}\right)$ and, for a constant $\varepsilon>0$,

$$
S_{\varepsilon}:=-\Delta+V_{\varepsilon} .
$$

Suppose that the following conditions are satisfied:

(i) For all $\varepsilon>0, S_{\varepsilon}$ is self-adjoint and bounded from below and $\sigma_{\mathrm{ess}}\left(S_{\varepsilon}\right) \subset[0, \infty)$.

(ii) There exists a nonempty open set $\Omega \subset\left\{x \in \mathbb{R}^{d} \mid V(x)<0\right\}$.

Then there exists a constant $\varepsilon_{0}>0$ such that, for all $\varepsilon \in\left(0, \varepsilon_{0}\right), S_{\varepsilon}$ has a discrete ground state.

Proof: By condition (ii), we can take a nonzero vector $f \in C_{0}^{\infty}(\Omega)$ (the set of infinitely differentiable functions on $\mathbb{R}^{d}$ with compact support in $\Omega$ ). Then it is easy to see that $\left\langle f_{\varepsilon}, S_{\varepsilon} f_{\varepsilon}\right\rangle$ $=\varepsilon^{-d}\left(a_{f} \varepsilon^{2}-\left|b_{f}\right|\right)$, where $a_{f}:=\nabla f \|^{2}, b_{f}=\langle f, V f\rangle<0$. Hence, taking $\varepsilon_{0}:=\sqrt{\left|b_{f}\right| / a_{f}}$ (note that $a_{f} \neq 0$ ), we have $\left\langle f_{\varepsilon}, S_{\varepsilon} f_{\varepsilon}\right\rangle<0$ for all $\varepsilon \in\left(0, \varepsilon_{0}\right)$. Hence, by the min-max principle and condition (i), $E_{0}\left(S_{\varepsilon}\right) \in \sigma_{\mathrm{d}}\left(S_{\varepsilon}\right)$.

Lemma 4.4: Let $V: \mathbb{R}^{d} \rightarrow \mathbb{R}$ be continuous on $\mathbb{R}^{d}$ with $\lim _{|\mathbf{x}| \rightarrow \infty} V(\mathbf{x})=0$. Suppose that $\Omega_{-}:=\left\{x \in \mathbb{R}^{d} \mid V(x)<0\right\} \neq \emptyset$. Then the following hold:

(i) $\quad-\Delta+V$ acting in $L^{2}\left(\mathbb{R}^{d}\right)$ is self-adjoint and bounded from below.

(ii) $\sigma_{\text {ess }}(-\Delta+V)=[0, \infty)$. 
(iii) $S_{\varepsilon}$ has a discrete ground state for all $\varepsilon \in\left(0, \varepsilon_{0}\right)$ with some $\varepsilon_{0}>0$.

Proof: Part (i) follows from the Kato-Rellich theorem. Part (ii) is proven by a simple application of Theorem XIII.15-(b) in Ref. 4.

Since $V$ is continuous, the set $\Omega_{-}$is open. Hence Lemma 4.3 implies the existence of a ground state of $S_{\varepsilon}$ for all $\varepsilon \in\left(0, \varepsilon_{0}\right)$ with some $\varepsilon_{0}>0$.

We consider a one-parameter family of Dirac operators,

$$
H_{\varepsilon}:=H_{0}+\frac{1}{\varepsilon} M\left(\beta \otimes 1_{2}\right) U_{F_{\varepsilon}}
$$

which is a scaled deformation of $H$.

Theorem 4.5: Assume Hypothesis (II) and (3.1). Suppose that $D_{3} \cos F$ is not identically zero. Then there exists a constant $\varepsilon_{0}>0$ such that, for all $\varepsilon \in\left(0, \varepsilon_{0}\right), H_{\varepsilon}$ has a discrete positive energy ground state or a discrete negative ground state.

Proof: We write $S_{ \pm}(F, M):=S_{ \pm}(F)$ to make explicit the dependence of $S_{ \pm}(F)$ on $M$. At least one of the sets $\left\{\mathbf{x} \in \mathbb{R}^{3} \mid\left(D_{3} \cos F\right)(\mathbf{x})>0\right\}$ and $\left\{\mathbf{x} \in \mathbb{R}^{3}\left(D_{3} \cos F\right)(\mathbf{x})<0\right\}$ is not empty. The function $D_{3} \cos F=-\left(D_{3} F\right) \sin F$ is bounded and continuous satisfying $\lim _{|\mathbf{x}| \rightarrow \infty}\left(D_{3} F\right)(\mathbf{x})=0$. Hence we can apply Lemma 4.4 to conclude that $S_{+}\left(F_{\varepsilon}, \varepsilon^{-1} M\right)$ or $S_{-}\left(F_{\varepsilon}, \varepsilon^{-1} M\right)$ has a discrete ground state for all $\varepsilon \in\left(0, \varepsilon_{0}\right)$ with some $\varepsilon_{0}>0$. This fact and Theorem 4.2 yield the desired result.

\section{SYMMETRY REDUCTION OF $\boldsymbol{H}$}

In this section, we show that, if $F$ is invariant under the rotations around the $x_{3}$ axis, then there exist infinitely many mutually orthogonal closed subspaces of $\mathcal{H}$ that reduce $H_{\varepsilon}$ for all $\varepsilon>0$ and each reduced part of $H_{\varepsilon}$ may have a positive energy ground state or a negative energy ground state. We use the cylindrical coordinates for points $\mathbf{x}=\left(x_{1}, x_{2}, x_{3}\right) \in \mathbb{R}^{3}$,

$$
x_{1}=r \cos \theta, \quad x_{2}=r \sin \theta, \quad x_{3}=z,
$$

where $\theta \in[0,2 \pi), r>0$. We assume the following.

Hypothesis (III): There exists a continuously differentiable function $G:(0, \infty) \times \mathbb{R} \rightarrow \mathbb{R}$ such that (i) $F(\mathbf{x})=G(r, z), \quad \mathbf{x} \in \mathbb{R}^{3} \backslash\{0\} ; \quad$ (ii) $\lim _{r+|z| \rightarrow \infty} G(r, z)=0 ; \quad$ (iii) $\sup _{r>0, z \in \mathbb{R}}(|\partial G(r, z) / \partial r|$ $+|\partial G(r, z) / \partial z|)<\infty$.

We take the vector field $\mathbf{n}$ to be of the form

$$
\mathbf{n}(\mathbf{x}):=(\sin \Theta(r, z) \cos (m \theta), \sin \Theta(r, z) \sin (m \theta), \cos \Theta(r, z)),
$$

where $\Theta:(0, \infty) \times \mathbb{R} \rightarrow \mathbb{R}$ is continuous and $m$ is a real constant.

Let $L_{3}:=-i x_{1} D_{2}+i x_{2} D_{1}$, the third component of the angular momentum. It is well known that $L_{3}$ is essentially self-adjoint on $C_{0}^{\infty}\left(\mathbb{R}^{3}\right)$. We denote its closure by the same symbol $L_{3}$. We set

$$
\Sigma_{3}:=\sigma_{3} \oplus \sigma_{3}
$$

acting on $\mathrm{C}^{4}$ and define

$$
K_{3}:=L_{3} \otimes 1_{2}+\frac{1}{2} \Sigma_{3} \otimes 1_{2}+\frac{m}{2} I \otimes \tau_{3},
$$

which is a self-adjoint operator acting in $\mathcal{H}$.

We denote by $T_{\varepsilon}(\varepsilon>0)$ the unitary dilation on $L^{2}\left(\mathbb{R}^{3}\right)$ with power $\varepsilon$,

$$
\left(T_{\varepsilon} f\right)(\mathbf{x}):=\varepsilon^{3 / 2} f(\varepsilon \mathbf{x}), \quad f \in L^{2}\left(\mathbb{R}^{3}\right), \text { a.e. } \mathbf{x} .
$$

Lemma 5.1: For all $\varepsilon>0, T_{\varepsilon} L_{3} T_{\varepsilon}^{-1}=L_{3}$. Hence $\left(T_{\varepsilon} \otimes 1_{2}\right) K_{3}\left(T_{\varepsilon} \otimes 1_{2}\right)^{-1}=K_{3}$ for all $\varepsilon>0$.

Proof: It is straightforward to see that, for all $f \in C_{0}^{\infty}\left(\mathbb{R}^{3}\right), T_{\varepsilon} L_{3} f=L_{3} T_{\varepsilon} f$. Since $C_{0}^{\infty}\left(\mathbb{R}^{3}\right)$ is a core of $L_{3}$, this equality extends to all $f \in D\left(L_{3}\right)$ showing that $L_{3} \subset T_{\varepsilon}^{-1} L_{3} T_{\varepsilon}$. The both sides are 
self-adjoint. Hence they coincide.

Lemma 5.2: Assume that

$$
\Theta(\varepsilon r, \varepsilon z)=\Theta(r, z), \quad(r, z) \in(0, \infty) \times \mathbb{R}, \quad \varepsilon>0 .
$$

Then, for all $t \in \mathbb{R}$ and $\varepsilon>0$, the operator equality

$$
e^{i t K_{3}} H_{\varepsilon} e^{-i t K_{3}}=H_{\varepsilon}
$$

holds.

Proof: We first prove (5.5) with $\varepsilon=1$. We have for all $t \in \mathbb{R}$,

$$
e^{i t K_{3}}=e^{i t L_{3}} e^{i t \Sigma_{3} / 2} \otimes e^{i t m \tau_{3} / 2}
$$

For all $f \in C_{0}^{\infty}\left(\mathbb{R}^{3}\right)$, we have

$$
\left(e^{i t L_{3}} f\right)(\mathbf{x})=f\left(x_{1} \cos t-x_{2} \sin t, x_{1} \sin t+x_{2} \cos t, z\right), \quad \mathbf{x} \in \mathbb{R}^{3} .
$$

Hence $e^{i t L_{3}}$ leaves $C_{0}^{\infty}\left(\mathbb{R}^{3}\right)$ invariant. It follows that, for all $f \in C_{0}^{\infty}\left(\mathbb{R}^{3} ; \mathrm{C}^{4}\right) \otimes \mathbb{C}^{2}, e^{i t K_{3}} f \in D\left(H_{0}\right)$ $=D(H)$ and

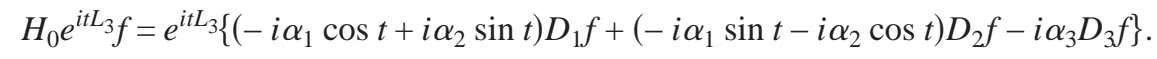

Using the matrix representation of $\alpha_{j}$, one can check that

$$
\alpha_{j} e^{i t \Sigma_{3} / 2}=e^{-i t \Sigma_{3} / 2} \alpha_{j} \quad(j=1,2), \quad\left[\alpha_{3}, e^{i t \Sigma_{3}}\right]=0 .
$$

It follows from these relations and (5.6),

$$
H_{0} e^{i t K_{3}} f=e^{i t K_{3}} H_{0} f
$$

We have

$$
\tau_{j} e^{i t m \tau_{3} / 2}=e^{i t m \tau_{3} / 2} \tau_{j} e^{i t m \tau_{3}} \quad(j=1,2), \quad \tau_{3} e^{i t m \tau_{3} / 2}=e^{i t m \tau_{3} / 2} \tau_{3}
$$

and

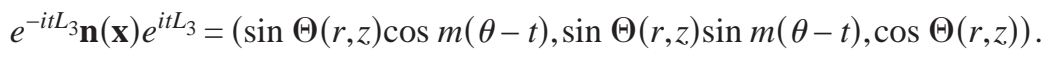

It follows from these relations that

$$
\beta \otimes 1_{2} U_{F} e^{i t K_{3}} f=e^{i t K_{3}}\left(\beta \otimes 1_{2}\right) U_{F} f .
$$

Combining (5.7) together with (5.8), we obtain $H e^{i t K_{3}} f=e^{i t K_{3}} H f$. Since $C_{0}^{\infty}\left(\mathbb{R}^{3} ; \mathrm{C}^{4}\right) \otimes \mathrm{C}^{2}$ is a core of $H$, this equality extends to all $f \in D(H)=D\left(H_{0}\right)$ showing $H \subset e^{-i t K_{3}} H e^{i t K_{3}}$. The both sides are self-adjoint. Thus (5.5) follows.

We next consider the case where $\varepsilon \neq 1$. We write $U_{F}=U(F, \mathbf{n})$. By Lemma 5.1, (5.8) and the fact that $T_{\varepsilon}$ is a bijection from $C_{0}^{\infty}\left(\mathbb{R}^{3}\right)$ onto itself, we have $\beta \otimes 1_{2} U\left(F_{\varepsilon}, \mathbf{n}_{\varepsilon}\right) e^{i t K_{3}} f=e^{i t K_{3}}(\beta$ $\left.\otimes 1_{2}\right) U\left(F_{\varepsilon}, \mathbf{n}_{\varepsilon}\right) f$. By condition (5.4), $\mathbf{n}_{\varepsilon}=\mathbf{n}$. Hence $\beta \otimes 1_{2} U\left(F_{\varepsilon}, \mathbf{n}_{\varepsilon}\right) e^{i t K_{3}} f=e^{i t K_{3}}\left(\beta \otimes 1_{2}\right) U\left(F_{\varepsilon}, \mathbf{n}\right) f$. Therefore (5.8) holds with $F$ replaced by $F_{\varepsilon}$. Thus, in the same way as in the preceding paragraph, one can prove (5.5).

We say that two self-adjoint operators on a Hilbert space strongly commute if their spectral measures commute.

Lemma 5.3: Assume (5.4). Then, for all $\varepsilon>0, H_{\varepsilon}$ and $K_{3}$ strongly commute.

Proof: It follows from Lemma 5.2 and the functional calculus for self-adjoint operators that $e^{i t K_{3}} e^{i s H_{\varepsilon}}=e^{i s H_{\varepsilon}} e^{i t K_{3}}$ for all $s, t \in \mathrm{R}$ and all $\varepsilon>0$. This implies the strong commutativity of $H_{\varepsilon}$ and $K_{3}$ (see Theorem VIII.13 in Ref. 6 for general criteria of the strong commutativity of self-adjoint operators).

Let 


$$
E:=(0, \infty) \times[0,2 \pi) \times \mathbb{R}=\{(r, \theta, z) \mid r>0, \theta \in[0,2 \pi), z \in \mathbb{R}\}
$$

and $\mathrm{d} \mu:=r \mathrm{~d} r \otimes \mathrm{d} \theta \otimes \mathrm{d} z$, a measure on $E$. Then one can define a unitary operator $Y: L^{2}\left(\mathbb{R}^{3}\right)$ $\rightarrow L^{2}(E, \mathrm{~d} \mu)$ by

$$
(Y f)(r, \theta, z):=f(r \cos \theta, r \sin \theta, z), \quad f \in L^{2}\left(\mathbb{R}^{3}\right) .
$$

For each $\ell \in \mathbb{Z}$, we define $\phi_{\ell}:[0,2 \pi) \rightarrow \mathrm{C}$ by

$$
\phi_{\ell}(\theta):=\frac{1}{\sqrt{2 \pi}} e^{i \ell \theta}, \quad \theta \in[0,2 \pi) .
$$

It is well known that $\left\{\phi_{\ell}\right\}_{\ell \in Z}$ is a complete orthonormal system of $L^{2}([0,2 \pi))$. For each $f \in L^{2}(E, \mathrm{~d} \mu)$, we define $\hat{f}:(0, \infty) \times \mathbb{Z} \times \mathbb{R}$ by

$$
\hat{f}(r, \ell, z):=\int_{0}^{2 \pi} \phi_{\ell}(\theta)^{*} f(r, \theta, z) \mathrm{d} \theta .
$$

We define an operator $D_{\theta}$ on $L^{2}(E, \mathrm{~d} \mu)$ as follows:

$$
\begin{gathered}
D\left(D_{\theta}\right):=\left\{\left.f \in L^{2}(E, \mathrm{~d} \mu)\left|\sum_{\ell=-\infty}^{\infty} \ell^{2} \int_{0}^{\infty} \mathrm{d} r r \int_{\mathbb{R}} \mathrm{d} z\right| \hat{f}(r, \ell, z)\right|^{2}<\infty\right\}, \\
\left(\widehat{D_{\theta} f}\right)(r, \ell, \theta)=i \ell \hat{f}(r, \ell, \theta), \quad f \in D\left(D_{\theta}\right) .
\end{gathered}
$$

Then $-i D_{\theta}$ is self-adjoint with

$$
\begin{gathered}
\sigma\left(-i D_{\theta}\right)=\sigma_{\mathrm{p}}\left(-i D_{\theta}\right)=\{\ell\}_{\ell \in \mathbb{Z}}=\mathbb{Z}, \\
\operatorname{ker}\left(-i D_{\theta}-\ell\right)=\left\{\left.g \phi_{\ell}\left|g:(0, \infty) \times \mathbb{R} \rightarrow \mathbb{C}, \int_{0}^{\infty} \mathrm{d} r r \int_{\mathbb{R}} \mathrm{d} z\right| g(r, z)\right|^{2}<\infty\right\} .
\end{gathered}
$$

It is not so hard to see that

$$
Y L_{3} Y^{-1}=-i D_{\theta}
$$

Hence

$$
\sigma\left(L_{3}\right)=\sigma_{\mathrm{p}}\left(L_{3}\right)=\mathbb{Z}
$$

Let

$$
\mathcal{M}_{\ell}:=\operatorname{ker}\left(L_{3}-\ell\right)=Y^{-1} \operatorname{ker}\left(-i D_{\theta}-\ell\right) .
$$

Then we have the orthogonal decomposition

$$
L^{2}\left(\mathbb{R}^{3}\right)=\oplus_{\ell=-\infty}^{\infty} \mathcal{M}_{\ell}, \quad L^{2}(E, \mathrm{~d} \mu)=\oplus_{\ell=-\infty}^{\infty} Y \mathcal{M}_{\ell} .
$$

By (5.13), we have

$$
\sigma\left(K_{3}\right)=\sigma_{\mathrm{p}}\left(K_{3}\right)=\left\{\ell+\frac{s}{2}+\frac{m t}{2} \mid \ell \in \mathbb{Z}, s= \pm 1, t= \pm 1\right\} .
$$

The eigenspace of $K_{3}$ with eigenvalue $\ell+(s / 2)+(m t / 2)$ is given by 


$$
\mathcal{M}_{\ell, s, t}:=\mathcal{M}_{\ell} \otimes \mathcal{C}_{s} \otimes \mathcal{T}_{t}
$$

under the natural identificaion $\mathcal{H}=L^{2}\left(\mathbb{R}^{3}\right) \otimes C^{4} \otimes C^{2}$, where $\mathcal{C}_{s}:=\operatorname{ker}\left(\Sigma_{3}-s\right)$ and $\mathcal{T}_{t}:=\operatorname{ker}\left(\tau_{3}-t\right)$. Then $\mathcal{H}$ has the orthogonal decomposition

$$
\mathcal{H}=\oplus_{\ell \in Z, s, t \in\{ \pm 1\}} \mathcal{M}_{\ell, s, t} .
$$

Lemma 5.3 implies the following fact.

Lemma 5.4: Assume (5.4). Then, for all $\varepsilon>0, H_{\varepsilon}$ is reduced by each $\mathcal{M}_{\ell, s, t}$.

We denote by $H_{\varepsilon}(\ell, s, t)$ by the reduced part of $H_{\varepsilon}$ to $\mathcal{M}_{\ell, s, t}$ and set

$$
H(\ell, s, t):=H_{1}(\ell, s, t)
$$

the reduced part of $H$ to $\mathcal{M}_{\ell, s, t}$.

For $s= \pm 1$ and $\ell \in \mathbb{Z}$, we define

$$
S_{s}(G, \ell):=-\frac{\partial^{2}}{\partial r^{2}}-\frac{1}{r} \frac{\partial}{\partial r}+\frac{\ell^{2}}{r^{2}}+\frac{\partial^{2}}{\partial z^{2}}+s M \frac{\partial \cos G}{\partial z}
$$

acting in $L^{2}((0, \infty) \times \mathbb{R}, r \mathrm{~d} r \mathrm{~d} z)$ with domain $D\left(S_{s}(G, \ell)\right):=C_{0}^{\infty}((0, \infty) \times \mathbb{R})$ and set

$$
\mathcal{E}_{0}\left(S_{s}(G, \ell)\right):=\inf _{f \in C_{0}^{\infty}((0, \infty) \times \mathbb{R}),\|f\|_{L^{2}((0, \infty) \times \mathrm{R}, r \mathrm{~d} r \mathrm{~d} z)=1}\left\langle f, S_{s}(G, \ell) f\right\rangle .}
$$

Theorem 5.5: Assume Hypothesis (III). Fix an $\ell \in \mathbb{Z}$ arbitrarily and $s= \pm 1$. Suppose that $\mathcal{E}_{0}\left(S_{s}(G, \ell)\right)<0$. Then, for each $t= \pm 1, H(\ell, s, t)$ has a discrete positive energy ground state or a discrete negative ground state.

Proof: Let

$$
\begin{gathered}
c_{\ell}:=\frac{1}{\sqrt{2 \pi}} \int_{0}^{2 \pi} \mathrm{d} \theta e^{-i \ell \theta} \cos (m \theta), \quad \mathrm{d}_{\ell}:=\frac{1}{\sqrt{2 \pi}} \int_{0}^{2 \pi} \mathrm{d} \theta e^{-i \ell \theta} \sin (m \theta), \\
n_{j, \ell}(r, z):=\left(\sin \Theta(r, z) c_{\ell}, \sin \Theta(r, z) d_{\ell}, \cos \Theta(r, z)\right), \\
\Phi_{G, \ell, t^{:}}=\cos G+i \sum_{j=1} n_{j, \ell} \sin G \otimes \tau_{j}+i t n_{3, \ell} \sin G, \\
D_{1, \ell}:=c_{\ell} \frac{\partial}{\partial r}-\frac{d_{\ell}}{r} \frac{\partial}{\partial \theta}, \quad D_{2, \ell}:=d_{\ell} \frac{\partial}{\partial r}+\frac{c_{\ell}}{r} \frac{\partial}{\partial \theta}
\end{gathered}
$$

and

$$
W_{G_{\varepsilon}, \ell, s, t}:=i \sum_{j=1}^{2} \sigma_{j} D_{j, \ell} \Phi_{G_{\varepsilon}, \ell, t}+i s D_{z} \Phi_{G_{\varepsilon}, \ell, t}, \quad \varepsilon>0 .
$$

Then we have

$$
\begin{aligned}
\left(Y \otimes 1_{2}\right) H_{\varepsilon}(\ell, s, t)^{2}\left(Y \otimes 1_{2}\right)^{-1}= & -\frac{\partial^{2}}{\partial r^{2}}-\frac{1}{r} \frac{\partial}{\partial r}+\frac{\ell^{2}}{r^{2}}+\frac{\partial^{2}}{\partial z^{2}}+\varepsilon^{-1} M\left(\begin{array}{c}
0 \\
W_{G_{\varepsilon}, \ell, s, t}
\end{array} W_{G_{\varepsilon}, \ell, s, t}^{*}\right)+M^{2} \\
= & : L_{\varepsilon}(\ell, s, t)+M^{2}
\end{aligned}
$$

on $C_{0}^{\infty}((0, \infty) \times \mathbb{R})$.

For each $f \in C_{0}^{\infty}((0, \infty) \times \mathbb{R})$ and $u_{t} \in \mathbb{C}^{2}$ satisfying $\|f\|=1,\left\|u_{t}\right\|=1$, and $\tau_{3} u_{t}=t u_{t}(t= \pm 1)$, we define 


$$
\begin{gathered}
\psi_{f}^{(1)}:=\left(f \otimes u_{t}, 0, \text { if } \otimes u_{t}, 0\right) \in \mathcal{M}(\ell, 1, t), \\
\psi_{f}^{(-1)}:=\left(0, f \otimes u_{t}, 0, \text { if } \otimes u_{t}\right) \in \mathcal{M}(\ell,-1, t) .
\end{gathered}
$$

Then we have

$$
\left\langle\psi_{f}^{(s)}, Y L_{1}(\ell, s, t) Y^{-1} \psi_{f}^{(s)}\right\rangle=2\left\langle f, S_{s}(F, \ell) f\right\rangle .
$$

By the present assumption, there exists a unit vector $f \in C_{0}^{\infty}((0, \infty) \times \mathbb{R})$ such that $\left\langle f, S_{s}(F, \ell) f\right\rangle$ $<0$. Note that $\sigma_{\text {ess }}\left(L_{1}(\ell, s, t)\right) \subset[0, \infty)$. Hence, by the min-max principle, $L_{1}(\ell, s, t)$ has a discrete ground state. This implies that $H(\ell, s, t)$ has a discrete positive energy ground state or a discrete negative ground state.

Theorem 5.6: Assume Hypothesis (III) and (5.4). Suppose that $\partial \cos G / \partial z$ is not identically zero. Then, for each $\ell \in \mathbb{Z}$, there exists a constant $\varepsilon_{\ell}>0$ such that, for all $\varepsilon \in\left(0, \varepsilon_{\ell}\right)$, each $H_{\varepsilon}(\ell, s, t)$ has a discrete positive energy ground state or a discrete negative ground state.

Proof: We write $S_{s, M}(F, \ell):=S_{s}(F, \ell)$ to make explicit the dependence of $S_{s}(F, \ell)$ on $M$. In the same way as in the proof of Theorem 4.5, one can take a vector $f_{\varepsilon} \in C_{0}^{\infty}((0, \infty) \times \mathbb{R})$ such that $\left\langle f_{\varepsilon}, S_{s, \varepsilon^{-1} M}\left(F_{\varepsilon}(\ell)\right) f_{\varepsilon}\right\rangle<0$ for all sufficiently small $\varepsilon>0$, where the smallness depends on $\ell$. It follows from the proof of the preceding theorem that $L_{\varepsilon}(\ell, s, t)$ has a discrete ground state.

Corollary 5.7: Assume Hypothesis (III) and (5.4). Suppose that $\partial \cos G / \partial z$ is not identically zero. Let $\varepsilon_{\ell}$ be as in Theorem 5.6 and, for each $N \in \mathbb{N}$ and $k>n(k, n \in \mathbb{Z}), \nu_{k, n}:=\min _{n+1 \leqslant \ell \leqslant k} \varepsilon_{\ell}$. Then, for each $\varepsilon \in\left(0, \nu_{k, n}\right), H_{\varepsilon}$ has at least $(k-n)$ discrete eigenvalues counting multiplicities.

Proof: We have $\sigma_{\mathrm{p}}\left(H_{\varepsilon}\right)=\cup_{\ell \in Z, s, t= \pm 1} \sigma_{\mathrm{p}}\left(H_{\varepsilon}(\ell, s, t)\right)$. By the preceding theorem, for each $\ell=n$ $+1, \ldots, k, H_{\varepsilon}(\ell, s, t)$ has a discrete eigenvalue. Thus the desired result follows.

Remark 5.1: This result is consistent with Theorem 3.2, because it reads in the present case

$$
N_{H_{\varepsilon}} \leqslant \frac{1}{\varepsilon^{4}} \frac{M^{2} C_{F}}{2 \pi^{2}}
$$

and the right-hand side diverges as $\varepsilon \rightarrow 0$.

\section{A UNITARY TRANSFORMATION}

In this section we show that, under Hypothesis (II), the Hamiltonian $H$ with $\mathbf{n}$ constant is unitarily equivalent to an operator which resembles a Dirac operator with a magnetic moment.

It is easy to see that the operator

$$
X_{F}:=\left(\begin{array}{cc}
e^{i F \otimes \tau \cdot \mathbf{n} / 2} & 0 \\
0 & e^{-i F \otimes \tau \cdot \mathbf{n} / 2}
\end{array}\right)
$$

is unitary. Under Hypothesis (II), we can define the following functions:

$$
B_{j}(\mathbf{x}):=\frac{1}{2} D_{j}(F(\mathbf{x}) \otimes \boldsymbol{\tau} \cdot \mathbf{n}(\mathbf{x})), \quad \mathbf{x} \in \mathbb{R}^{3}, j=1,2,3 .
$$

We set

$$
\mathbf{B}:=\left(B_{1}, B_{2}, B_{3}\right)
$$

and introduce

$$
H(\mathbf{B}):=H_{0}+M \beta-\boldsymbol{\sigma} \cdot \mathbf{B}
$$

acting in $\mathcal{H}$. Note that, under Hypothesis (II), the operator $-\boldsymbol{\sigma} \cdot \mathbf{B}$ is a bounded self-adjoint operator. Hence, by a simple application of the Kato-Rellich theorem, $H(\mathbf{B})$ is self-adjoint with $D(H(\mathbf{B}))=D\left(H_{0}\right)$.

Proposition 6.1: Assume Hypothesis (II) and that $\mathbf{n}$ is constant. Then 


$$
X_{F} H X_{F}^{-1}=H(\mathbf{B}) .
$$

Proof: Noting the fact that $(\boldsymbol{\tau} \cdot \mathbf{n})^{2}=1_{2}$, we have

$$
\Phi_{F}=e^{i F \otimes \boldsymbol{\tau} \cdot \mathbf{n}} .
$$

It follows from this fact and (3.8) that $X_{F} H X_{F}^{-1} \psi=H(\mathbf{B}) \psi$ for all $\psi \in\left[\oplus^{4} C_{0}^{\infty}\left(\mathbb{R}^{3}\right)\right] \otimes \mathbb{C}^{2}$. Since $\left[\oplus^{4} C_{0}^{\infty}\left(\mathbb{R}^{3}\right)\right] \otimes \mathbb{C}^{2}$ is a core of $H(\mathbf{B})$, the operator equality (6.5) follows.

\section{ACKNOWLEDGMENT}

The authors thank Dr. T. Miyao for discussions.

${ }^{1}$ N. Sawado, Phys. Lett. B 524, 289 (2002).

${ }^{2}$ H. Kalf and O. Yamada, J. Math. Phys. 42, 2667 (2001).

${ }^{3}$ B. Thaller, The Dirac Equation (Springer, Berlin, Heidelberg, 1992).

${ }^{4}$ M. Reed and B. Simon, Methods of Modern Mathematical Physics IV: Analysis of Operators (Academic, New York, 1978).

${ }^{5}$ M. Reed and B. Simon, Methods of Modern Mathematical Physics II: Fourier Analysis, Self-Adjointness (Academic, New York, 1975).

${ }^{6}$ M. Reed and B. Simon, Methods of Modern Mathematical Physics I: Functional Analysis (Academic, New York, 1972). 